\title{
Switching the Localized Surface Plasmon Resonance of Single Gold Nanorods with a Phase-Change Material and the Implementation of a Cellular Automata Algorithm Using a Plasmon Particle Array
}

\author{
Takashi Hira, Takayuki Uchiyama, Kenta Kuwamura, Yuya Kihara, \\ Tasuku Yawatari, and Toshiharu Saiki \\ Department of Electronics and Electrical Engineering, Keio University, 3-14-1 Hiyoshi, Kohoku, Yokohama 223-8522, Japan \\ Correspondence should be addressed to Toshiharu Saiki; saiki@elec.keio.ac.jp
}

Received 20 August 2014; Revised 9 October 2014; Accepted 9 October 2014

Academic Editor: Takashi Yatsui

Copyright (C) 2015 Takashi Hira et al. This is an open access article distributed under the Creative Commons Attribution License, which permits unrestricted use, distribution, and reproduction in any medium, provided the original work is properly cited.

We investigate the modulation of the localized surface plasmon resonance (LSPR) of a gold nanorod (AuNR) using a GeSbTe film as an active medium. We demonstrate high-contrast switching of LSPR in an AuNR/GST/Au thin film sandwich structure upon phase change. To go beyond this single-particle switching functionality, we consider a plasmon particle system interacting with a phase-change material (PCM) to discuss the possibility of parallel processing devices with memory functionality, exploiting the plasticity and threshold behavior that are inherent characteristics of PCMs. We demonstrate that the temporal and spatial evolution of a plasmon-PCM array system can be equivalent to a cellular automata algorithm.

\section{Introduction}

Control of localized surface plasmon resonance (LSPR) excited on metal nanostructures has drawn attention for applications in dynamic switching and for tuning the functionality of plasmonic optical devices [1,2]. Basically, control of LSPR relies on its sensitivity to the optical permittivity of the environment due to dielectric screening and mode hybridization [3, 4]. As reversible active media for LSPR control, chalcogenide phase-change materials (PCMs) [5] such as $\mathrm{GeSbTe}$ are promising for the development of high-contrast robust plasmonic switching devices [6-8] owing to significant differences in the dielectric constants of their crystalline and amorphous phases, as well as their stable plasticity at room temperature and their rapid crystallization (within several tens of nanoseconds). More importantly, GeSbTe can be nonthermally amorphized on a subpicosecond timescale with femtosecond laser pulse excitation $[9,10]$. By exploiting these attractive properties of GeSbTe, we have demonstrated the LSPR switching of individual Au nanospheres on a GeSbTe thin film by alternating irradiation by a femtosecond pulse laser for amorphization and a continuous wave (cw) laser for crystallization [11].

The metal-dielectric-metal nanosandwich structure is an effective approach for enhancing LSPR switching contrast. When two metal particles are coupled, their dipoles are hybridized and split into symmetric and antisymmetric modes. The latter mode is called a magnetic dipole mode, in which the dipole moments of the two particles are aligned in opposite directions and generate a current loop [12-15]. The LSPR peak positions of the hybridized modes, particularly the magnetic dipole mode, shift dramatically with the refractive index of the dielectric material owing to the retardation effect.

Owing to the plasticity and the threshold behavior during both amorphization and crystallization of PCMs, PCMbased LSPR switching elements possess a dual functionality of memory and processing. Integration of LSPR switching elements so that they interact with each other will allow us to build a new computing device. In this paper, as a specific demonstration of this idea, we discuss the implementation of a cellular automata (CA) algorithm $[16,17]$ into interacting LSPR switching elements. CA is used as a tool for 


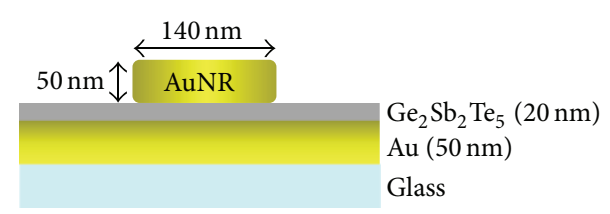

(a)

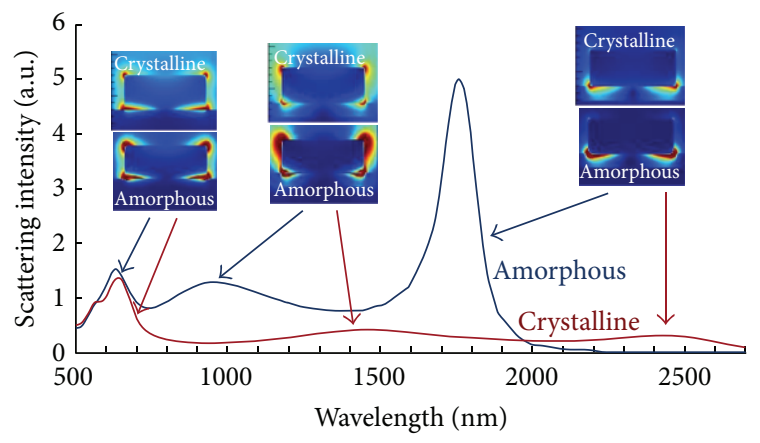

(b)

FIGURE 1: (a) Model of the AuNR/GST/Au nanosandwich structure for numerical calculation. (b) Numerically calculated scattering spectra from an AuNR/GST/Au nanosandwich for crystalline and amorphous GST. The insets show the electric field distributions in the vicinity of the AuNR for the three dominant bands.

the modeling of complex and dynamic systems, including natural and social phenomena. Any CA system is composed of cells with a finite number of states, and each cell interacts with neighboring cells. The interaction is local, with the next state of a cell being a function of the current state of itself and its neighbors. In the model we propose that PCM cells, which can be in one of two states (amorphous and crystalline), are allowed to interact with each other by being linked by a gold nanorod (AuNR), whose LSPR peak wavelength is determined by the phase of PCM cells on either side. The CA program proceeds by irradiating with a light pulse train. The local rule set is defined by the temperature rise in the PCM cells induced by the LSPR of the Au nanorod, which is subject to the intensity and wavelength of the irradiating pulse.

\section{FDTD Simulation}

Figure 1(a) illustrates the layered configuration of the Au and $\mathrm{Ge}_{2} \mathrm{Sb}_{2} \mathrm{Te}_{5}$ (GST) sandwich structure. Numerical simulations based on the finite-difference time-domain (FDTD) method were conducted to determine the appropriate wavelength at which to monitor the LSPR switching of the AuNR. A scattering spectrum of the AuNR was calculated under broadband plane wave illumination at normal incidence. The spatial discretization was set to $1 \mathrm{~nm}$, and the dielectric constants of $\mathrm{Au}$ and GST were obtained from $[18,19]$, respectively. Figure 1(b) shows scattering spectra from the AuNR with crystalline and amorphous phases of the GST layer. The spectrum consists of three dominant bands around 600,1000, and $1800 \mathrm{~nm}$ for amorphous GST and 600, 1500, and $2500 \mathrm{~nm}$ for crystalline GST.

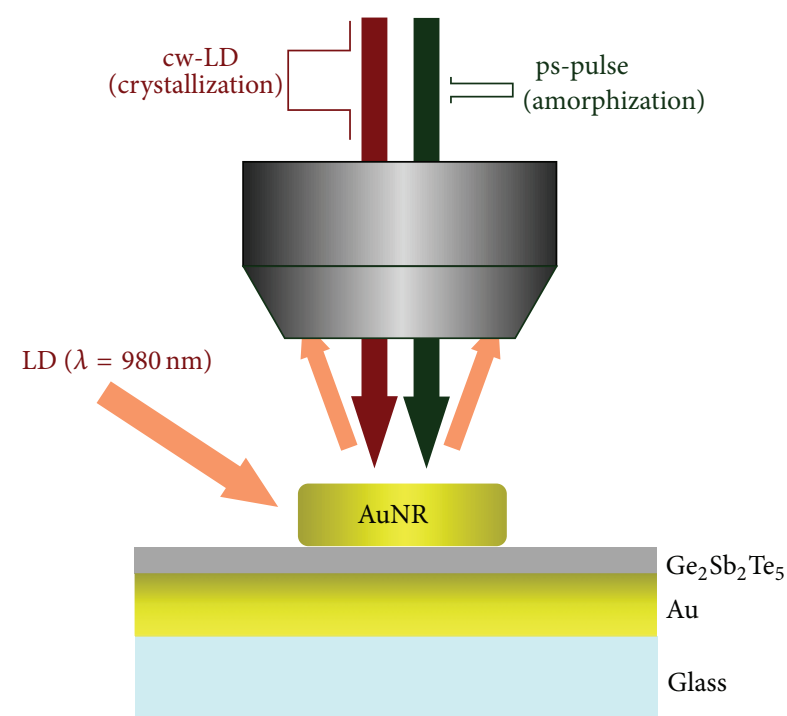

FIGURE 2: Experimental setup for dark-field scattering measurement of single Au nanorods.

The electric field distribution (Figure 1(b) inset) confirms that the lowest bands come from interactions between the AuNR and the underlying Au layer. As in the nanosandwich, this band originates from antisymmetric coupling between the AuNR dipole and its image in the Au layer. The magnetic field intensity distribution (not shown) provides support for this peak assignment; the antisymmetric mode generates a magnetic moment due to the induced electrical dipoles, which oscillate out-of-phase to create an effective current loop.

The LSPR peak position and broadening were significantly altered upon phase change $(\Delta \lambda \approx 500 \mathrm{~nm}$ for the intermediate band and $\Delta \lambda \approx 700 \mathrm{~nm}$ for the lowest band). The higher refractive index of the crystalline phase than that of the amorphous phase causes a larger retardation effect, and thus the LSPR occurs at a longer wavelength. The broader LSPR for crystalline GST is due to the larger imaginary component of the GST dielectric constant, which causes a stronger damping of the LSPR. A high switching contrast is expected around 1000 and $1800 \mathrm{~nm}$, so the former band was selected to demonstrate this, and the scattering intensity ratio of the AuNR for GST in the amorphous phase to that of GST in the crystalline phase is larger than 5.

\section{LSPR Switching of a Single Gold Nanorod}

After the deposition of a 50-nm-thick Au thin film on a glass substrate, a 20 -nm-thick GST film was formed by sputtering. A colloidal solution of AuNRs ( $140 \mathrm{~nm}$ long, $50 \mathrm{~nm}$ in diameter) was used, and an AuNR dispersion in ethanol was drop-cast onto the GST film. The substrate was heated to rapidly evaporate the ethanol and prevent the aggregation of AuNRs.

The optical setup for single-AuNR spectroscopy is illustrated in Figure 2. To amorphize the GST, a picosecond pulse from a diode-pumped solid-state laser $(\lambda=532 \mathrm{~nm}$; pulse 


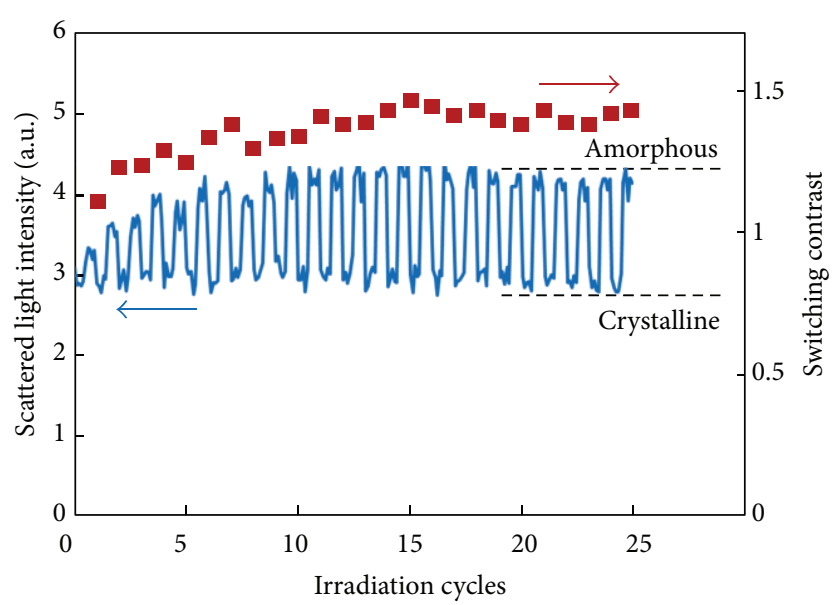

Figure 3: Time trace of scattered light intensity $(\lambda=980 \mathrm{~nm})$ from AuNR/GST/Au nanosandwich demonstrating repeatable modulation of LSPR band by alternate irradiation with femtosecond pulsed laser for amorphization and cw LD for crystallization. The switching contrast for each cycle is also plotted.

width: 300 ps) was delivered through a microscope objective. The pulse fluence was approximately $200 \mathrm{~mJ} / \mathrm{cm}^{2}$. To induce crystallization, a laser diode (LD) with $\lambda=830 \mathrm{~nm}$ was used under $\mathrm{cw}$ operation. To monitor the switching behavior of a single AuNR at an LSPR band around $1000 \mathrm{~nm}$, the AuNR was illuminated by a LD with $\lambda=980 \mathrm{~nm}$ in dark-field configuration, and the scattered light was detected through the objective.

Figure 3 shows a time trace of the scattered light intensity. Starting with the GST layer in the crystalline phase, amorphization and crystallization were repeated under alternating irradiation by picosecond laser pulses and the cw LD. LSPR switching with good stability and repeatability was observed. Here, we define the switching contrast, $I_{a} / I_{c}$, where $I_{a}$ and $I_{c}$ are the scattered light intensities for substrates in the amorphous and crystalline phases, respectively. The obtained switching contrast of 1.5 was smaller than expected on the basis of the numerical simulations. This may be due to residual background scattered light originating from the roughness of the GST film surface. The amorphization process may be localized, occurring only in a small volume just below the AuNR, where the electric field enhancement occurs. However, we assumed in the simulation that the GST was fully amorphized throughout the entire volume. This could also explain the deviation from the simulation results.

In the initial stage of the switching operation, a gradual increase in the modulation contrast was observed. Although it was difficult to determine what was occurring from the optical measurements alone, one possible explanation is as follows. The modification of the surface morphology due to volume expansion of the GST film may be different after each amorphization process. There is a volume expansion of approximately $10 \%$ upon amorphization from crystalline GST [20]. Therefore, there could be a slight change in the position or configuration of the AuNR in the initial stage that eventually becomes stabilized.

\section{Implementation of Cellular Automata Algorithm}

We attempted to implement a CA [16, 17] algorithm into the interacting LSPR switching elements. A CA consists of a uniform array of identical cells in which each cell has a finite number of states and interacts with a few neighboring cells. A rule set is provided to define how each cell is updated at each new time step as a result of its interactions. The rule set may involve some physical mechanism that takes account of local interactions.

One very simple CA is a one-dimensional line of cells, each existing in one of two states ( 0 or 1$)$. The local transition rule for each cell is defined as a function of the adjacent two neighbors. Figure 4 illustrates a one-dimensional CA composed of plasmon particles and a phase change material. $\mathrm{GeSbTe}$ nanopads are arranged in a regular array on an $\mathrm{Au}$ film. Each nanopad corresponds to a cell in the CA. Some pads are in the crystalline phase (state " 0 "), while others are in the amorphous phase (state "1"). In order to enable interaction between neighboring nanopads, they are coupled to each other by AuNRs. When the system is uniformly illuminated with laser light at a specific wavelength, some nanorods resonate with the light while others do not, depending on whether the nanopads on both sides are amorphous or crystalline. If a nanorod resonates with the light, the nanopads on both sides absorb more light and are heated, and eventually a phase change may take place depending on what temperature the nanopad reaches.

An electromagnetic simulation was performed to calculate the temperature increase in the nanopads under light irradiation at different wavelengths. The model unit element of the CA used for this calculation is illustrated in Figure 5(a). After solving for the electric field distribution using the FDTD method, we calculated the absorbed power density $\left(Q_{\mathrm{abs}}\right)$ distribution within the nanopad, employing the imaginary part of the permittivity of crystalline and amorphous GST. Figure 5(b) shows the temperature of nanopads in different configurations as a function of irradiation wavelength. Here, we assume that the temperature increase in a nanopad due to light absorption is proportional to the heat generated, which can be obtained by integrating $Q_{\mathrm{abs}}$ over the volume of the nanopad. The irradiation light intensity was determined such that the maximum temperature of a nanopad in the crystalline phase exceeded the melting point of GST $\left(\sim 600^{\circ} \mathrm{C}\right)$. For example, if the wavelength is $1800 \mathrm{~nm}$ and the two nanopads adjacent to an $\mathrm{Au}$ nanorod are in the amorphous phase, the temperature will reach $350^{\circ} \mathrm{C}$. Furthermore, if one pad is amorphous and the other is crystalline, the temperature of the crystalline nanopad will increase to $950^{\circ} \mathrm{C}$, while the other only reaches $70^{\circ} \mathrm{C}$.

In a one-dimensional CA (array of nanopads), the temperature of each nanopad can be calculated in a similar fashion. The laser irradiation conditions are generally different for amorphization and crystallization. However, although somewhat hypothetical, if we assume an irradiation light pulse with a width longer than the crystallization time and with a fall time much shorter than the crystallization time, the pulse can induce both amorphization and crystallization, 


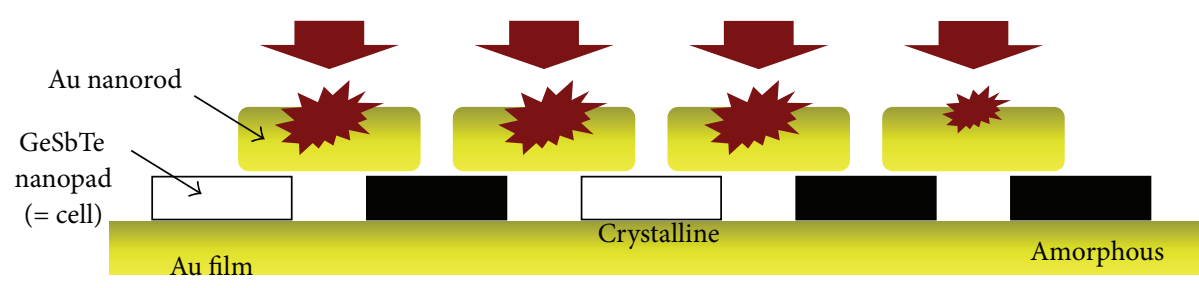

FIGURE 4: One-dimensional CA model of GeSbTe nanopads. The nanopads are coupled to each other by Au nanorods.

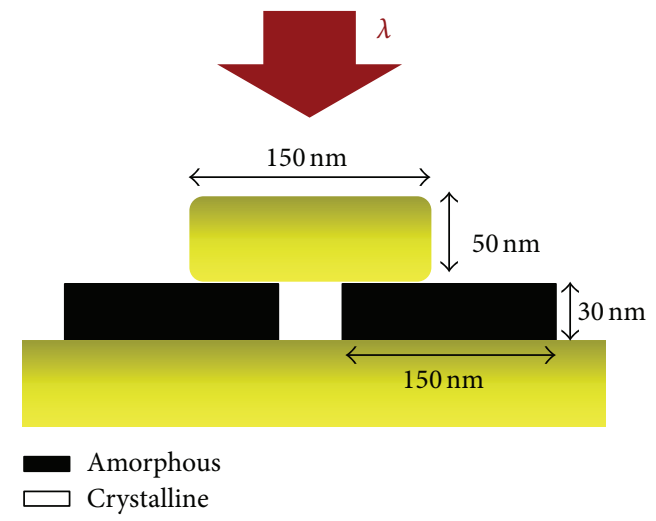

(a)

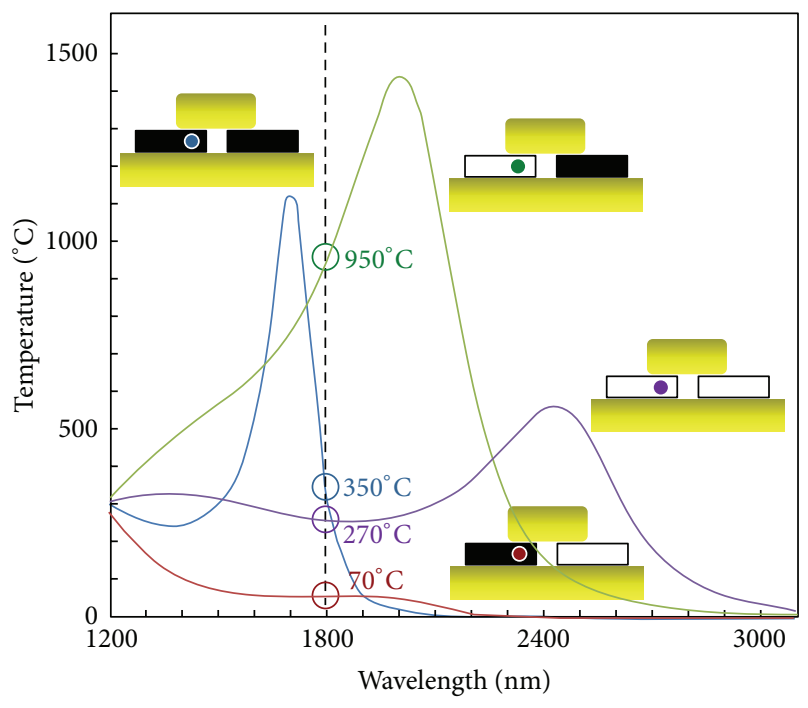

(b)

FIGURE 5: (a) Model for electromagnetic and thermal simulations. (b) Maximum temperature of the nanopads as a function of irradiation wavelength.

depending solely on the temperature rise. To determine the states (crystalline or amorphous) for the subsequent time step, we make the following simple assumptions. (1) If the temperature exceeds the melting point of GST, the crystalline phase will become amorphous. (2) Between the crystallization temperature $\left(\sim 150^{\circ} \mathrm{C}\right)$ and the melting point, the amorphous phase will become crystalline. (3) Below the crystallization temperature, nothing will happen. Under

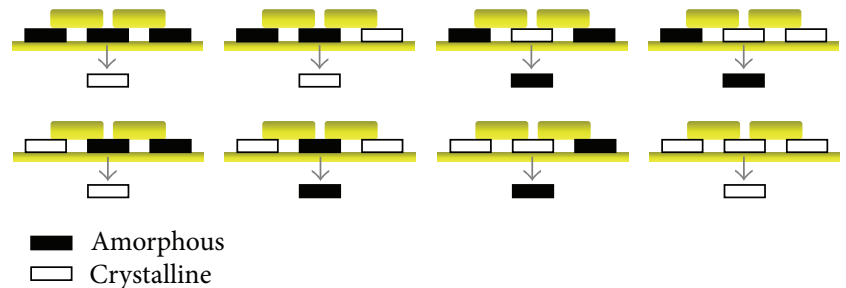

FIGURE 6: Rule number 54 for one-dimensional array of cellular automata.

these assumptions, at an irradiation wavelength of $1800 \mathrm{~nm}$, we obtained the rule set illustrated in Figure 6, called rule number 54 . When the irradiation wavelength is shifted, we can obtain another rule set.

\section{Conclusion}

Single-particle spectroscopy of an Au nanosandwich, in which a thin GST layer is sandwiched between interacting AuNRs and an Au film, was conducted. The LSPR band structure was determined using the FDTD method, and its physical causes were discussed. A very large band shift was obtained for the hybridized modes between the AuNR and Au film upon phase change of the GST layer. An experimental demonstration of LSPR modulation was performed for the LSPR band around $1000 \mathrm{~nm}$, at which a switching contrast of $>5$ would be expected. Stable and repeatable switching with a contrast of 1.5 was obtained. Finally, as an application of high-contrast LSPR switching, the implementation of a onedimensional cellular automata algorithm using Au nanorod and GST nanopad array was discussed.

\section{Conflict of Interests}

The authors declare that there is no conflict of interests regarding the publication of this paper.

\section{Acknowledgments}

The authors are grateful to T. Shintani for providing them with GST thin film samples. This work was supported by JSPS KAKENHI Grant no. 24226006 and partially by Advanced Photon Science Alliance Project from MEXT. 


\section{References}

[1] Y. Leroux, J. C. Lacroix, C. Fave et al., "Active plasmonic devices with anisotropic optical response: a step toward active polarizer," Nano Letters, vol. 9, no. 5, pp. 2144-2148, 2009.

[2] N. A. Mirin, K. Bao, and P. Nordlander, "Fano Resonances in plasmonic nanoparticle aggregates," Journal of Physical Chemistry A, vol. 113, no. 16, pp. 4028-4034, 2009.

[3] M. Pelton, J. Aizpurua, and G. Bryant, "Metal-nanoparticle plasmonics," Laser and Photonics Reviews, vol. 2, no. 3, pp. 136159, 2008.

[4] W. A. Murray and W. L. Barnes, "Plasmonic materials," Advanced Materials, vol. 19, no. 22, pp. 3771-3782, 2007.

[5] M. Wuttig and N. Yamada, "Phase-change materials for rewriteable data storage," Nature Materials, vol. 6, no. 11, pp. 824-832, 2007.

[6] Z. L. Sámson, K. F. MacDonald, F. de Angelis et al., "Metamaterial electro-optic switch of nanoscale thickness," Applied Physics Letters, vol. 96, no. 14, Article ID 143105, 2010.

[7] Z. L. Sámson, S.-C. Yen, K. F. MacDonald et al., "Chalcogenide glasses in active plasmonics," Physica Status Solidi-Rapid Research Letters, vol. 4, no. 10, pp. 274-276, 2010.

[8] Y. G. Chen, T. S. Kao, B. Ng et al., "Hybrid phase-change plasmonic crystals for active tuning of lattice resonances," Optics Express, vol. 21, no. 11, pp. 13691-13698, 2013.

[9] M. Konishi, H. Santo, Y. Hongo, K. Tajima, M. Hosoi, and T. Saiki, "Ultrafast amorphization in $\mathrm{Ge}_{10} \mathrm{Sb}_{2} \mathrm{Te}_{13}$ thin film induced by single femtosecond laser pulse," Applied Optics, vol. 49, no. 18, pp. 3470-3473, 2010.

[10] J. Takeda, W. Oba, Y. Minami, T. Saiki, and I. Katayama, "Ultrafast crystalline-to-amorphous phase transition in $\mathrm{Ge}_{2} \mathrm{Sb}_{2} \mathrm{Te}_{5}$ chalcogenide alloy thin film using single-shot imaging spectroscopy," Applied Physics Letters, vol. 104, no. 26, Article ID 261903, 4 pages, 2014.

[11] T. Hira, T. Homma, T. Uchiyama, K. Kuwamura, and T. Saiki, "Switching of localized surface plasmon resonance of gold nanoparticles on a GeSbTe film mediated by nanoscale phase change and modification of surface morphology," Applied Physics Letters, vol. 103, no. 24, Article ID 241101, 2013.

[12] T. Pakizeh, M. S. Abrishamian, N. Granpayeh, A. Dmitriev, and M. Käll, "Magnetic-field enhancement in gold nanosandwiches," Optics Express, vol. 14, no. 18, pp. 8240-8246, 2006.

[13] A. Dmitriev, T. Pakizeh, M. Käll, and D. S. Sutherland, "Goldsitica-gold nanosandwiches: tunable bimodal plasmonic resonators," Small, vol. 3, no. 2, pp. 294-299, 2007.

[14] Y. Ekinci, A. Christ, M. Agio, O. J. F. Martin, H. H. Solak, and J. F. Löffler, "Electric and magnetic resonances in arrays of coupled gold nanoparticle in-tandem pairs," Optics Express, vol. 16, no. 17, pp. 13287-13295, 2008.

[15] G. Lévêque and O. J. F. Martin, "Optical interactions in a plasmonic particle coupled to a metallic film," Optics Express, vol. 14, no. 21, pp. 9971-9981, 2006.

[16] S. Wolfram, "Statistical mechanics of cellular automata," Reviews of Modern Physics, vol. 55, no. 3, pp. 601-644, 1983.

[17] S. Wolfram, "Cellular automata as models of complexity," Nature, vol. 311, no. 5985, pp. 419-424, 1984.

[18] P. B. Johnson and R. W. Christy, "Optical constants of the noble metals," Physical Review B, vol. 6, no. 12, pp. 4370-4379, 1972.

[19] K. Shportko, S. Kremers, M. Woda, D. Lencer, J. Robertson, and M. Wuttig, "Resonant bonding in crystalline phase-change materials," Nature Materials, vol. 7, no. 8, pp. 653-658, 2008.
[20] V. Weidenhof, I. Friedrich, S. Ziegler, and M. Wuttig, "Atomic force microscopy study of laser induced phase transitions in $\mathrm{Ge}_{2} \mathrm{Sb}_{2} \mathrm{Te}_{5}$," Journal of Applied Physics, vol. 86, no. 10, pp. 58795887, 1999. 

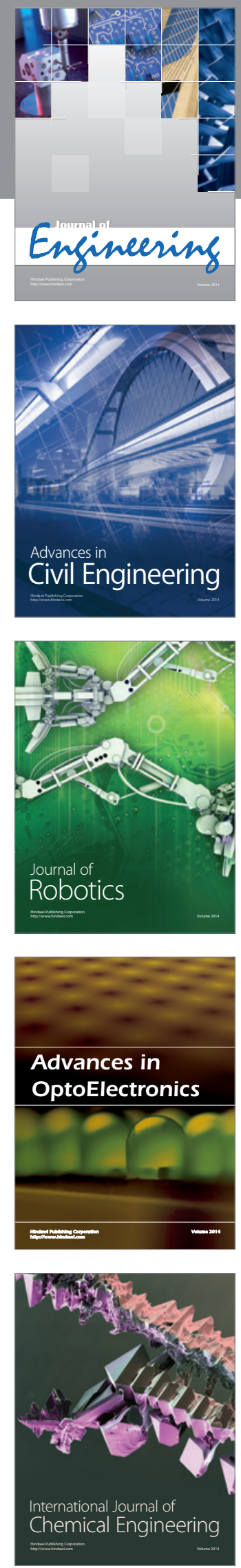

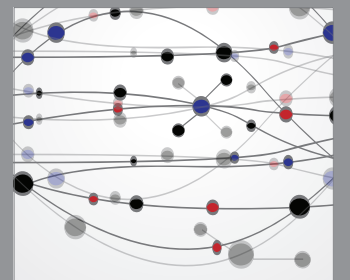

The Scientific World Journal
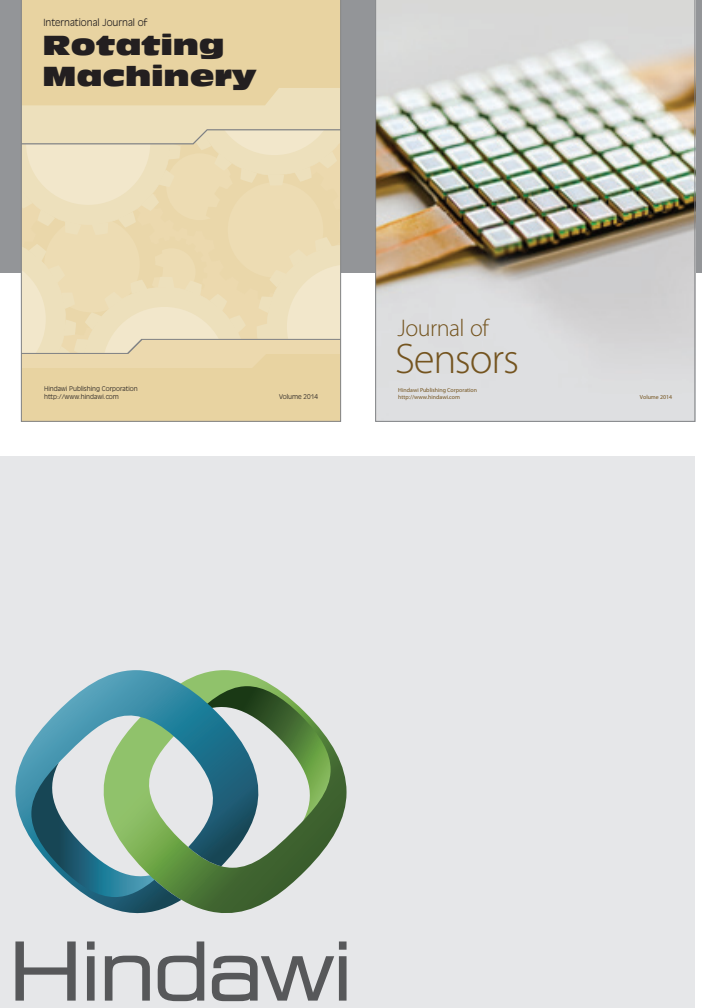

Submit your manuscripts at http://www.hindawi.com
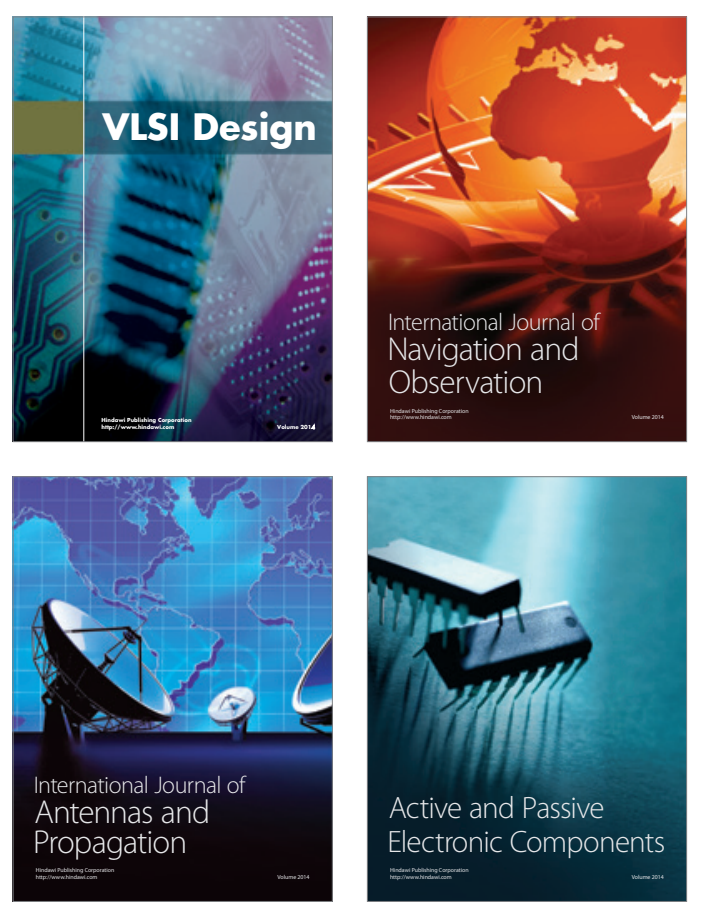
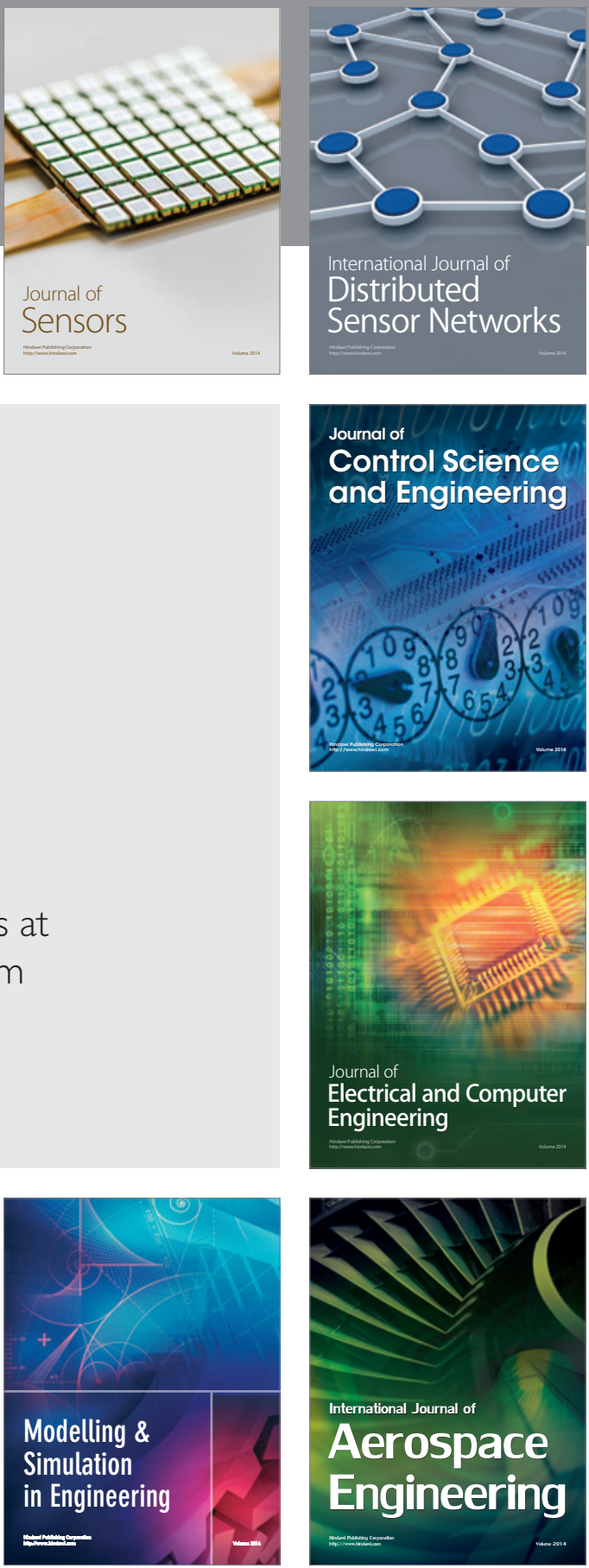

Journal of

Control Science

and Engineering
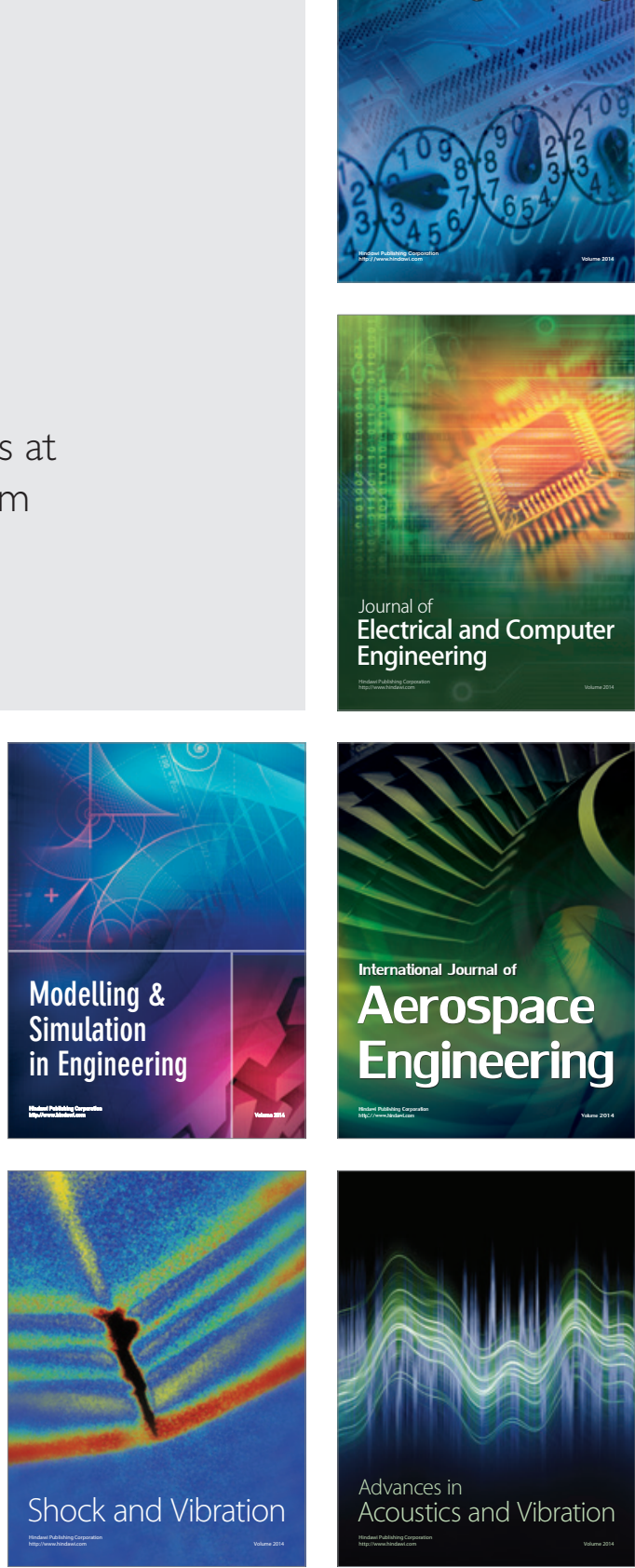